\title{
Synchronization and multi-mode dynamics of mutually coupled semiconductor lasers
}

\author{
Claudio R. Mirasso ${ }^{1,2}$, Miroslav Kolesik ${ }^{1,3}$, Marcelo Matus ${ }^{1}$, J.K. White ${ }^{4}$, and Jerome V. Moloney ${ }^{1}$ \\ 1 Arizona Center for Mathematical Science, University of Arizona, Tucson, AZ 85721. \\ 2 Departament de Física, Universitat de les Illes Balears, E-07071 Palma de Mallorca, Spain \\ ${ }^{3}$ Institute of Physics, Slovak Academy of Sciences, Bratislava, Slovakia. \\ ${ }^{4}$ Nortel Networks, High Performance Optical Solutions, 3500 Carling Ave. Ottawa ON K2H 8E9, Canada.
}

\begin{abstract}
Dynamics of coupled semiconductor lasers is investigated by numerical simulations. A realistic laser simulation engine is used to study the synchronization and dynamical regime in two mutually coupled Fabry-Perot and/or DFB lasers. Both, single- and multi-mode operation regimes are studied with emphasis on the role of the multiple laser-cavity modes. Our findings indicate that the two laser synchronize within each laser-cavity mode, while the synchronization across different cavity modes is significantly weaker.
\end{abstract}

Synchronization between coupled nonlinear oscillators has recently attracted attention of many researchers. A rich palette of behaviors has been observed in a wide variety of systems including, among others, population dynamics, coupled neurons, and lasers [1 5]. The interest in the synchronization between chaotic semiconductor lasers has been motivated by its potential for practical applications for example in communication systems using chaos to camouflage the transmitted messages [6,7]. In most of the cases the coupling between the subsystems includes a delay that accounts for the time the information takes to travel from on subsystem to the other. This delay introduces additional degrees of freedom to the system and leads to a qualitative different dynamics. The effect of the delay between two mutually coupled semiconductor lasers has been studied recently [4, 8] in a regime of long delays and moderate injection couplings. A spontaneous symmetry breaking was observed together with a retarded synchronization of chaotic regimes between the two subsystems. Similar studies, but with weak couplings and short delay times, have demonstrated localized synchronization of relaxation oscillations [12].

A semiconductor laser model, described by partial differential equations and including parabolic gain model, was used for the first time in ref. [5] to study feedback effects. In the latter paper, it was shown that weak external feedback can promote multi-longitudinal mode instabilities in an otherwise nominally single mode semiconductor laser, a fact that was also observed experimentally [9]. Moreover, in ref. [5] it is found that when two identical semiconductor lasers subjected to optical feedback are coupled unidirectionally, the same individual laser cavity mode could synchronize to its counterpart eventhough the others modes might be out of synchronization. Despite the relevance that multimode behaviour may have in some cases, majority of numerical simulations have been carried out with the rate equations model that assumes a single laser-cavity mode operation and neglect spatial dependencies. Although the agreement between the rate-equation based models and experimental observations is very good in general, questions concerning the role of the multi-mode laser operation arise [9]. Though it is possible to extend the Lang-Kobayashi-type models to multi-mode systems [10], we feel that the underlying approximations are difficult to control and prefer a more direct approach. The present work aims at further understanding of the multi-mode behavior in mutually coupled lasers by numerical simulations that are free of the usual rate-equation model approximations. To this end, we use a laser simulator with a full spatial and temporal resolution [11]. The simulation engine allows us to perform realistic numerical experiments on systems consisting of various types of semiconductor lasers as well as passive cavities and coupling between the subsystems.

We consider two identical devices, which will be either pairs of Fabry-Perot or DFB lasers. We pump both lasers with the same injection current very close to their solitary threshold. The distance between the two lasers is set to $1.2 \mathrm{~m}$, or equivalently a flight time for the light of $\tau \sim 4 \mathrm{~ns}$. A neutral density filter reduces the coupling between the lasers, which we fix to a value of $6 \%$ of transmission. For the Fabry-Perot laser we consider devices of $250 \mu \mathrm{m}$ length and $4 \mu \mathrm{m}$ width with natural, as-cleaved, facet reflectivities. In the case of DFB lasers, we use slightly longer devices $(400 \mu \mathrm{m})$ with simple Bragg gratings, i.e. without grating phase inserts, with a coupling coefficient of 5000 $\mathrm{m}^{-1}$ and zero reflectivity at the facets. Though such devices exhibit two symmetric grating supported modes, due to the asymmetry induced by the coupling with the counterpart laser, one of the modes is greatly suppressed. We choose the parameters of both types of lasers such that they operate with carrier densities at which the active layer exhibits the alpha factor around 3 .

The simulational model includes full many-body microscopic gain and refractive index and correctly accounts for gain dispersion in a broad frequency band. The flexibility of the simulator allows us to consider both Fabry-Perot type and DFB type of lasers without any restrictions of their modal properties. The simulator also accounts for both mutual injection and feedback coming from the front facet of the counterparting laser. Moreover, the simulator also allows us to check situations in which the optical feedback has negligible effects, as reported in [4], [12], but we can 
anticipate that the results do not qualitatively change. Under these conditions we approach as much as possible the situation reported in recent experiments [4], [12].

The main features we observe in the simulations with the Fabry-Perot laser twins can be summarized as follow:

1. We observe a LFF behavior, characterized by a sudden drop of the total intensity, similar to the one reported in refs. [4], [8]. This behavior resembles the well known LFF regime that appears in the case of a laser subjected to optical feedback (see for example [13] and references therein). However, we have observed that this regime persists even when we exclude feedback effects from the facet of the other laser, which is an indication that mutual injection alone may induce this kind of instabilities.

2. We observe a well defined leader-laggard dynamics, as reported experimentally and numerically with a rate equation model [4, [8], where the role of the leader and laggard changes randomly from one dropout to the other.

3. We observe a high degree of synchronization between the total output power of both lasers when one of the outputs is shifted with respect to the other by a time $\tau, \tau$ being the time it takes the light to fly from one laser to the other.

4. We only observe significant degree of synchronization if one of the series is shifted with respect to the other by an integer, but odd, multiple of $\tau$.

In Figure 1 we show the typical time traces of the total output power and the optical spectra of both lasers, the latter being averaged over a whole LFF cycle. In panel a) the output power of both laser exhibit the LFF features that we have already mentioned. As expected, fast irregular pulsations, in the GHz range, develop within these slow LFF cycle. In panel b) it can be seen that the lasers operate in a multi-mode regime. Despite this complicated dynamics, the spectra of both lasers are so similar to each other that it is very difficult to distinguish them. This is an indication of synchronization between the two lasers. However, these spectra do not tell us much about the dynamical evolution of the individual longitudinal modes. To gain insight into this problem we concentrate on the dynamics that take place within the different longitudinal modes of the FP lasers. To resolve the modes, we use a Fabry-Perot filter with a FHWHM bandwidth of $10 \mathrm{GHz}$ that allows us to isolate each individual longitudinal laser-cavity mode. In Figure 2 we plot the temporal evolution of one of the main mode's power for both lasers for a time interval that corresponds to the range $\sim 100-200$ ns of panel a) of Figure 1. When one of the series is shifted by $\tau$ a well-synchronized dynamics can be observed.

To characterize quantitatively the degree of synchronization between the different longitudinal modes of the two lasers, we compute the cross correlation function between the same longitudinal mode of the two lasers, defined as:

$$
S_{i}(\Delta t)=\frac{<\delta P_{1}^{i}(t) \delta P_{2}^{i}(t-\Delta t)>}{\sqrt{<\left(\delta P_{1}^{i}(t)\right)^{2}><\left(\delta P_{2}^{i}(t)\right)^{2}>}}
$$

where $P_{1}^{i}(t)$ and $P_{2}^{i}(t)$ represent the output power of the i-th longitudinal mode of each laser. Figure 3 shows the cross correlation function between the total power, the power of one of the main modes (located at $\sim-200 \mathrm{GHz}$ in figure $1 \mathrm{~b}$ )) and the power of one side mode (in this case the one located at $\sim-600 \mathrm{GHz}$ in figure $1 \mathrm{~b}$ )). In all the cases we observe maxima of the cross correlation function at $\pm \tau$. In addition, we also observe correlation, although smaller, at $\pm 3 \tau, \pm 5 \tau$, etc. On the other hand, when computing the cross correlation function between different longitudinal laser-cavity modes, we observe almost no correlation as can be seen in Fig. 3, panel d) for the mode located at $\sim-200 \mathrm{GHz}$ in one laser and the one located at $\sim-70 \mathrm{GHz}$ in the other laser. This fact indicates that the synchronization takes place only between the same longitudinal modes of the two lasers while the correlation between different longitudinal modes is rather weak. The fact that the same longitudinal mode of the different lasers synchronize was also observed in a system of two unidirectionally coupled semiconductor lasers [5].

As in the experiments and previous numerical simulations [4], [8] we also observe synchronization at sub-nanosecond time scale. However, the quality of the synchronization depends on the bandwidth of the detector. In Figure 4 we plot the correlation coefficient, or the value of the cross correlation function calculated at a time $\tau$, vs. the bandwidth of the detector for both the main mode (dashed line) and the total intensity (solid line). The synchronization is better for the individual longitudinal modes than for the total intensity and it extends almost over the whole range of detection without loosing its quality. The partial loss of synchronization in the slower-detector regime is due to the fact that the actual wave forms emitted by the lasers consist of trains of rather short pulses that are blurred when the detector response time is longer than the typical pulse duration. On the other hand, the synchronization gets worse for very fast detector as well. This is because of the lack of synchronization between different laser-cavity modes and by interference effects between them. As can be noted in the figure, the detection bandwidth for the isolated 
longitudinal mode is restricted to frequencies up to $10 \mathrm{GHz}$ due to of the previous optical filtering process. In any case, it is important to remark that a a high degree of synchronization is obtained for a wide detection bandwidth.

Finally, we considered a similar situation that we have already discussed but the lasers are now two DFB lasers. They are placed at the same distance and pumped close to threshold. The observed behavior of the output power is qualitatively similar to the one showed in Figure 1 a). However, we observe in the optical spectra that the lasers operate mainly in one longitudinal mode and only one side mode carries a small fraction of power. As in the FP case, the spectra of both lasers are very similar to each other, indicating a high degree of synchronization. After filtering the longitudinal modes we compute again the cross correlation function. In Figure 5 we plot this function for the two modes and for the total power. As expected, there are only small differences between the cross correlation of the total power and the one of the main longitudinal mode. But it can be also seen that the side modes synchronize to its counterpart at the same time shift $\pm \tau, \pm 3 \tau$, etc. as the total power or the main mode power do. This again indicates that the synchronization takes place at the same mode of the different lasers.

The important difference from the Fabry-Perot system case is that with the DFB lasers we can identify the laser mode that is responsible for the LFF behavior. Moreover, we can directly check if the other mode, the suppressed one, plays any role in the destabilization process. In Fig. 6 we plot, for comparison, the time-dependent modal powers during two consecutive dropouts. It is important to point out that for the time traces we have a time resolution of $\sim$ 0.1 ps. It can be clearly seen that the side mode typically exhibits measurable power only after a power dropout of the main mode develops. After the main mode recovers, the side-mode power steadily decreases until the next dropout, increasing the side-mode suppression ratio to several orders of magnitude. That is a strong indication that the side mode is actually not important for the LFF behavior and does not play any role in triggering the power dropouts. By the same token, it is also a strong indication that the single-mode models actually do capture the essential physics of the phenomenon.

In conclusion we have carried out a study of the dynamics of two distant, mutually coupled semiconductor lasers. To describe the lasers we have used a laser simulator with full spatial and temporal resolution that captures correctly the dynamics of both, Fabry-Perot and DFB lasers and includes a realistic model for the active medium. We have observed synchronization between the two output powers when one of the series is shifted with respect to the other by a time $\tau$ corresponding to the external cavity length. By filtering individual laser-cavity modes we have observed that this synchronization takes place between the the same individual longitudinal mode of the two lasers. On the other hand, the degree of synchronization between different laser-cavity modes turns out to be much smaller. As a consequence, the quality of the synchronization is better for the individual longitudinal modes than for the total power. We have also studied coupled DFB lasers to compare a multi-mode regime with an essentially single-mode situation. Our findings indicate that the dynamics responsible for the LFF behavior and the output power synchronization takes place within a single laser-cavity mode. Moreover, we have also observed that the suppressed mode does not play any role in triggering the $\mathrm{LFF}$.

This work was funded by Spanish MCyT under projects TIC99-0645-C05-02 and BFM2000-1108, by DGES under project PB97-0141-C02-01 and also by AFOSR grant no. F4962-00-1-0002 and AFOSR DURIP grant no. F4962-001-0190. M.K. was partly supported by the GASR grant VEGA 2/7174/20.

[1] S.H. Strogatz and I. Stewart, Sci.Am. 269, 68 (1993).

[2] U. Ernst, K. Pawelzik, and T. Geisel, Phys.Rev.Lett. 74, 1570 (1995).

[3] R. Roy and K.S. Thornburg, Jr., Phys.Rev.Lett. 72, 2009 (1994); T. Sugawara, M. Tachikawa, T. Tsukamoto, and T. Shimizu, Phys.Rev.Lett. 72, 3502 (1994); K. Otsuka, R. Kawai, S.L. Hwong, J.Y. Ko, and J.L. Chern, Phys.Rev.Lett. 84, 3049 (2000).

[4] T. Heil, I. Fischer, W. Elssser, J. Mulet and C.R. Mirasso, Phys. Rev. Lett. 86, 795 (2001).

[5] J.K. White, and J.V. Moloney, Phys. rev. A 59, 2422 (1999).

[6] G.D. VanWiggeren and R. Roy, Science 279, 1198 (1998); C.R. Mirasso, P. Colet, and P. Garcia-Fernandez, IEEE Photon.Tech.Lett 8, 299 (1996); J.P. Goedgebuer, L. Larger, and H. Porte, Phys.Rev.Lett. 80, 2249 (1998).

[7] I. Fischer, Y. Liu, and P. Davis, Phys.Rev. A 62, 011801(R) (2000).

[8] J. Mulet, C. Mirraso, T. Heil, and I. Fisher, Physics and simulation of optoelectronic devices IX, SPIE Proc., to be published.

[9] G. Vaschenko, M. Guidici, J.J. Rocca, C.S. Menoni, J.R. Tredicce, and S. Balle, Phys. Rev. Lett. 81, 5536 (1998).

[10] E.A. Viktorov, and P. Mandel Phys. Rev. Lett. 85, 3157 (2000); J. K. White, "Communication with chaotic semiconductor lasers", Ph.D. Dissertation, The University of Arizona (1999). 
[11] M. Kolesik, and J.V. Moloney, IEEE J. Quantum Electron. , to be published.

[12] A. Hohl, A. Gavrielides, T. Erneux, and V. Kovanis, Phys.Rev.Lett. 78, 4745 (1997); A. Hohl, A. Gavrielides, T. Erneux, and V. Kovanis. Phys. Rev. Lett. A 59, 3941 (1999).

[13] G. H. M. van Tartwijk and D. Lenstra, Quantum Semiclass. Opt. 7, 87 (1995). 


\section{FIGURE CAPTIONS}

Figure 1- Output intensity (with a detection bandwidth of $0.5 \mathrm{GHz}$ ) of the two coupled Fabry-Perot lasers (a), and their time-averaged optical spectra (b).

Figure 2- Synchronization of the output powers in the most-intense laser-cavity mode for both lasers. One of the outputs (showed in dashed line) is delayed by the external cavity trip time $\tau$.

Figure 3- Cross-correlation function of the output powers of the Fabry-Perot laser twins in the a) total output, b) most intense laser-cavity mode, and c) one of the weak side modes. Panel d) shows the cross-correlation between different laser cavity modes, with no significant synchronization present.

Figure 4- Correlation coefficient as a function of the detector bandwidth. The full curve (and star symbols) corresponds to the total output power, while the dashed line (and diamond symbols), that lasts until $10 \mathrm{GHz}$, shows the result for a single, filtered laser-cavity mode.

Figure 5- Cross-correlation function of the output powers of the two coupled DFB lasers. Panels a) and b) show the total output powers and the main mode correlations, respectively. Panel c) shows the cross-correlation functions between the side-modes of the two lasers.

Figure 6- Output power of the dominant mode (upper curve) and of the side mode (lower curve) of one of the DFB laser. 

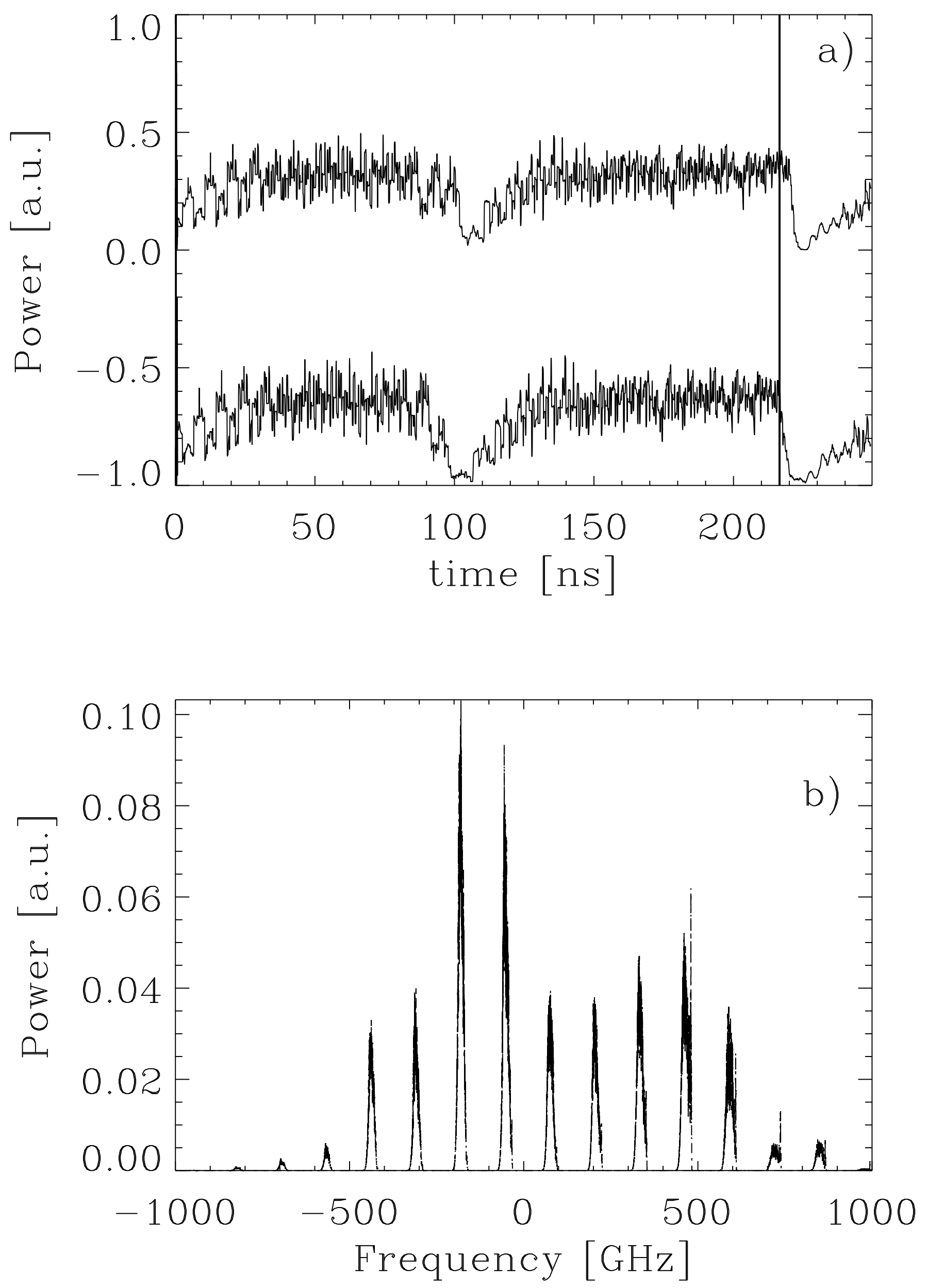


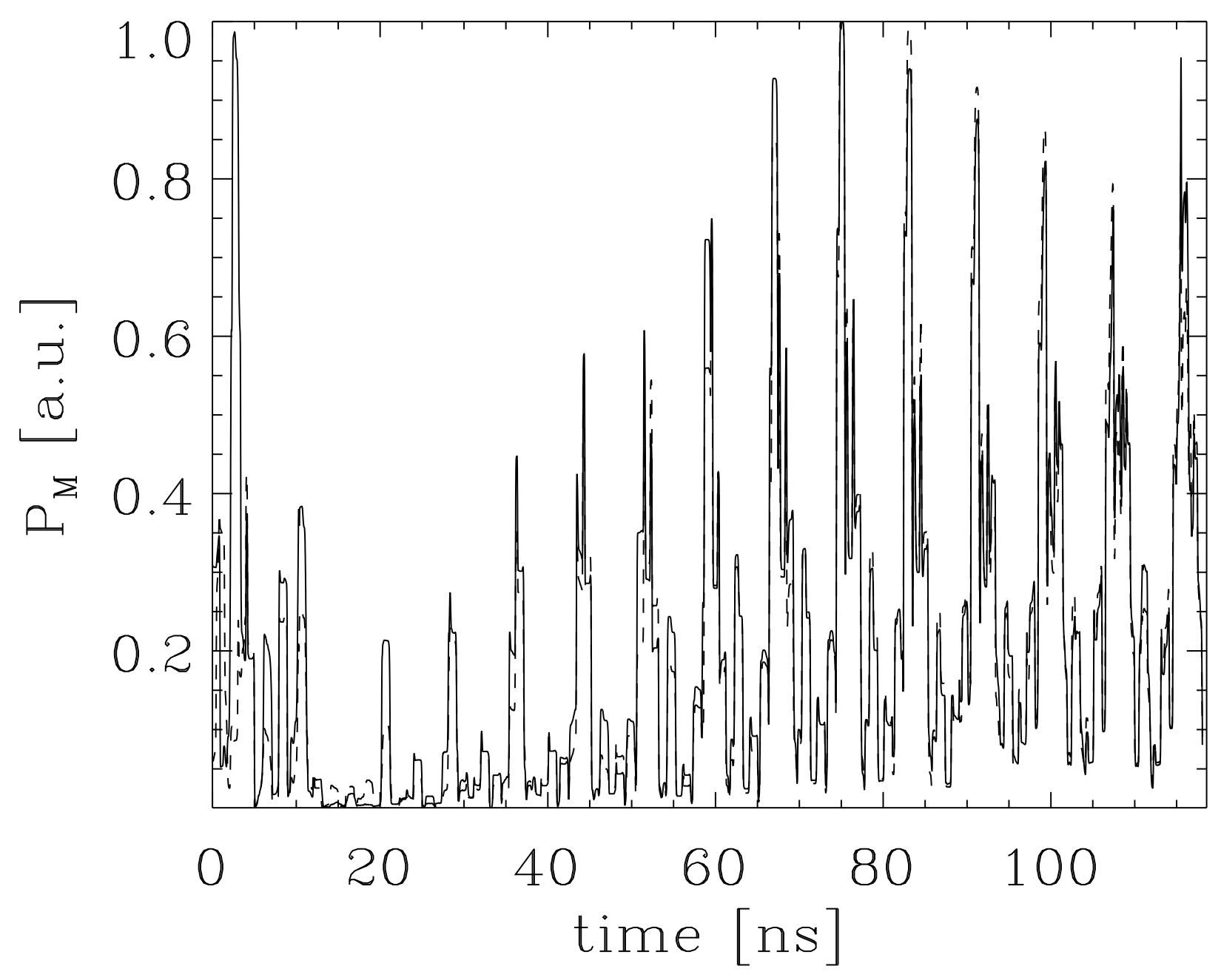




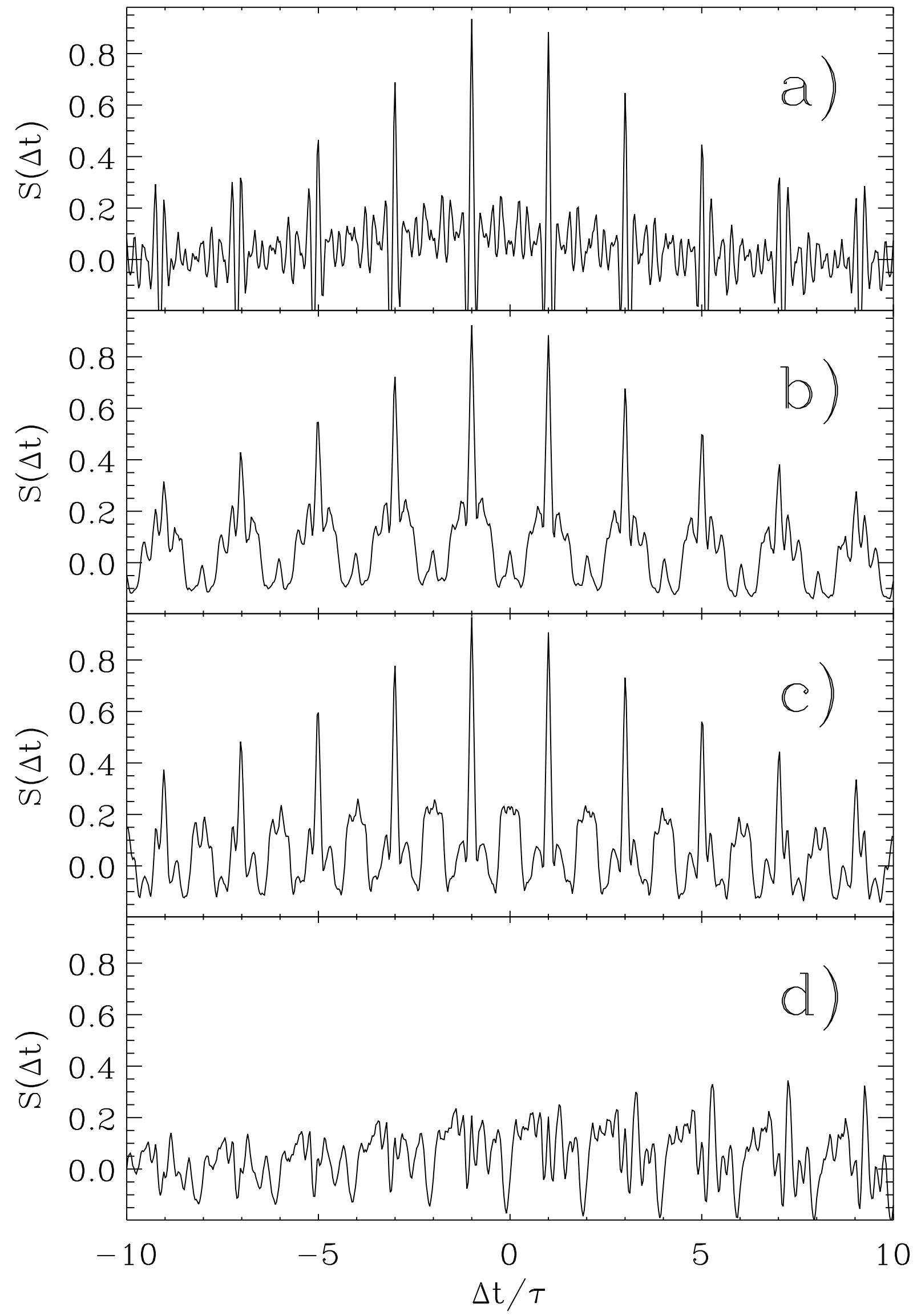




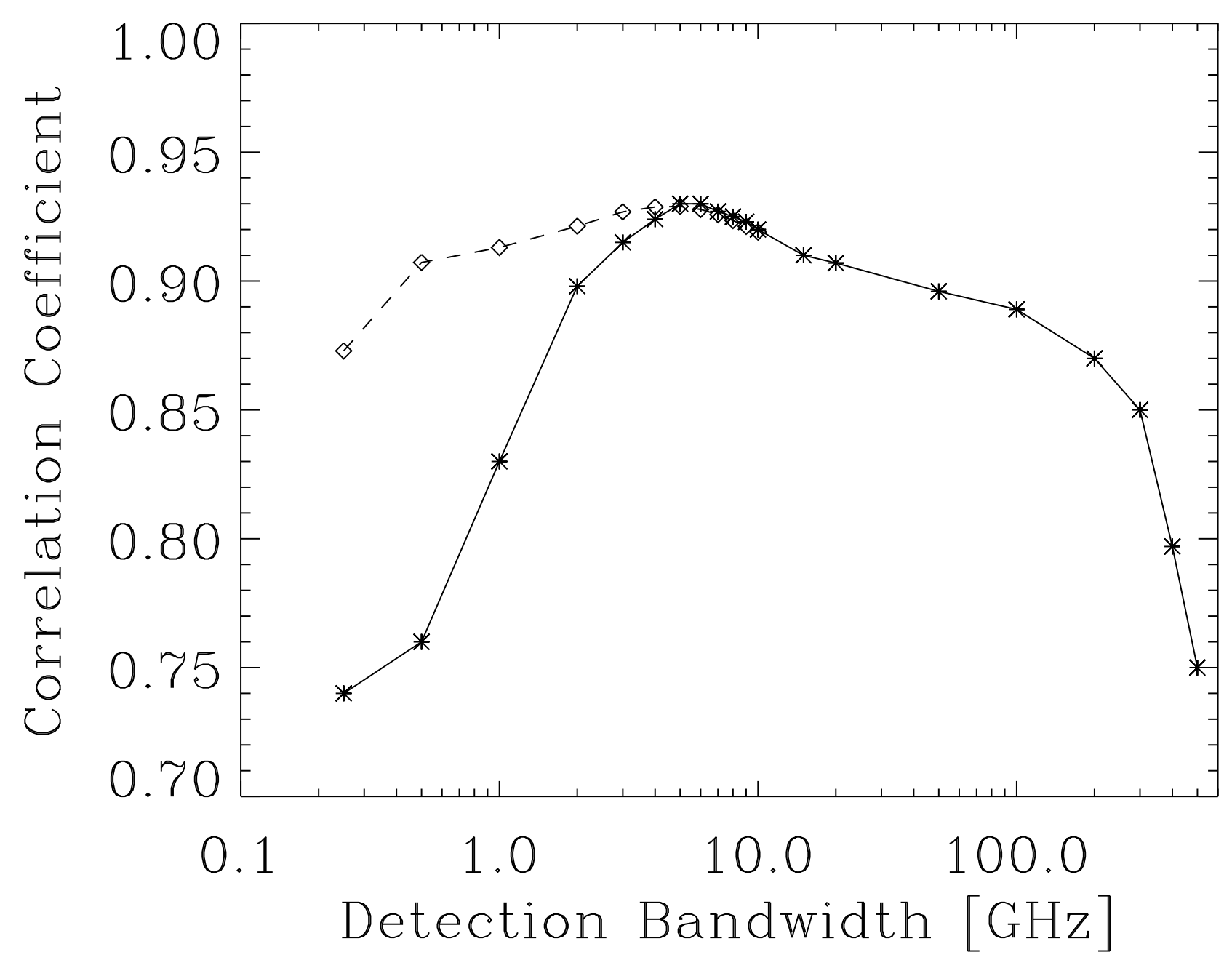




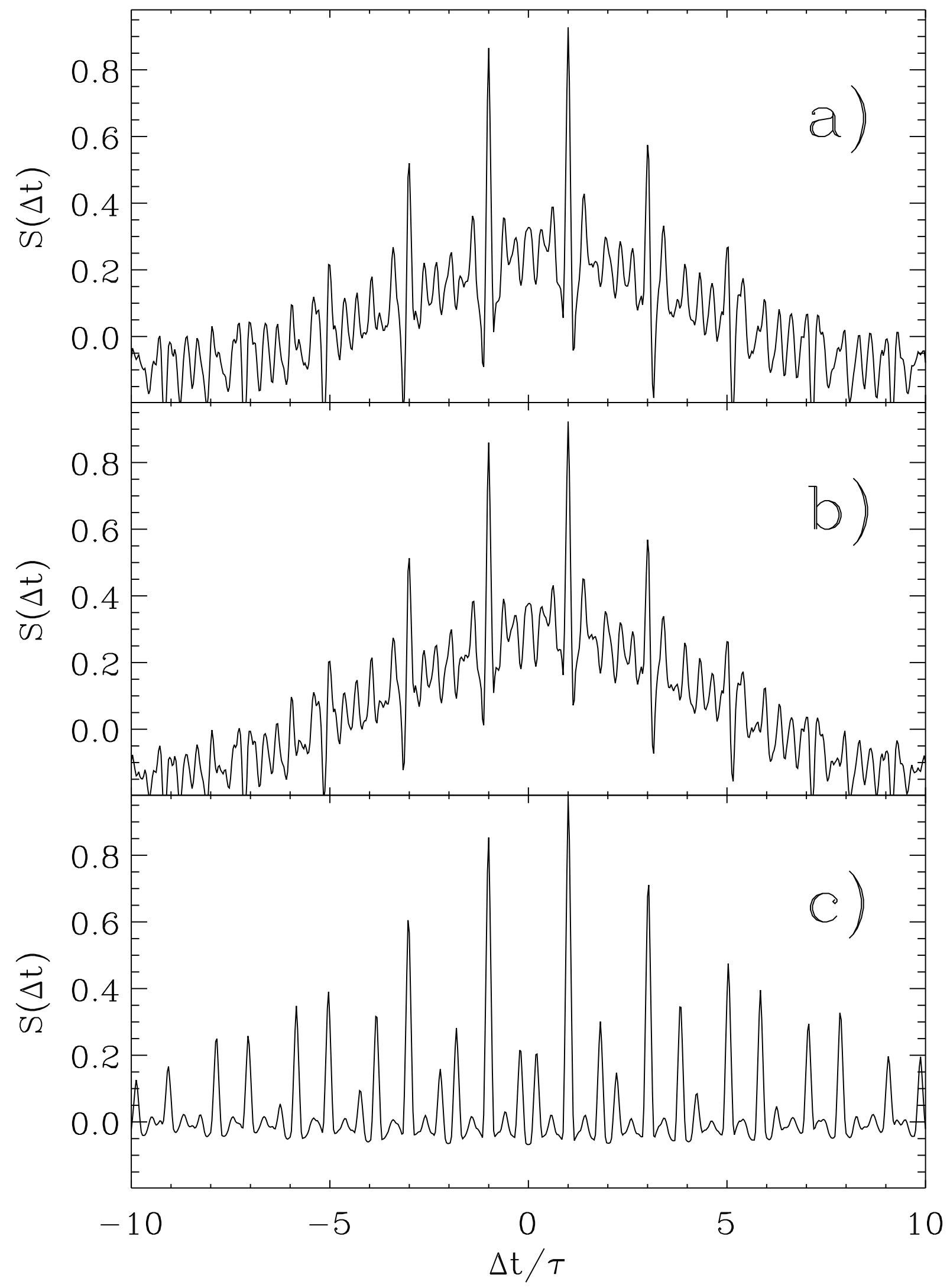




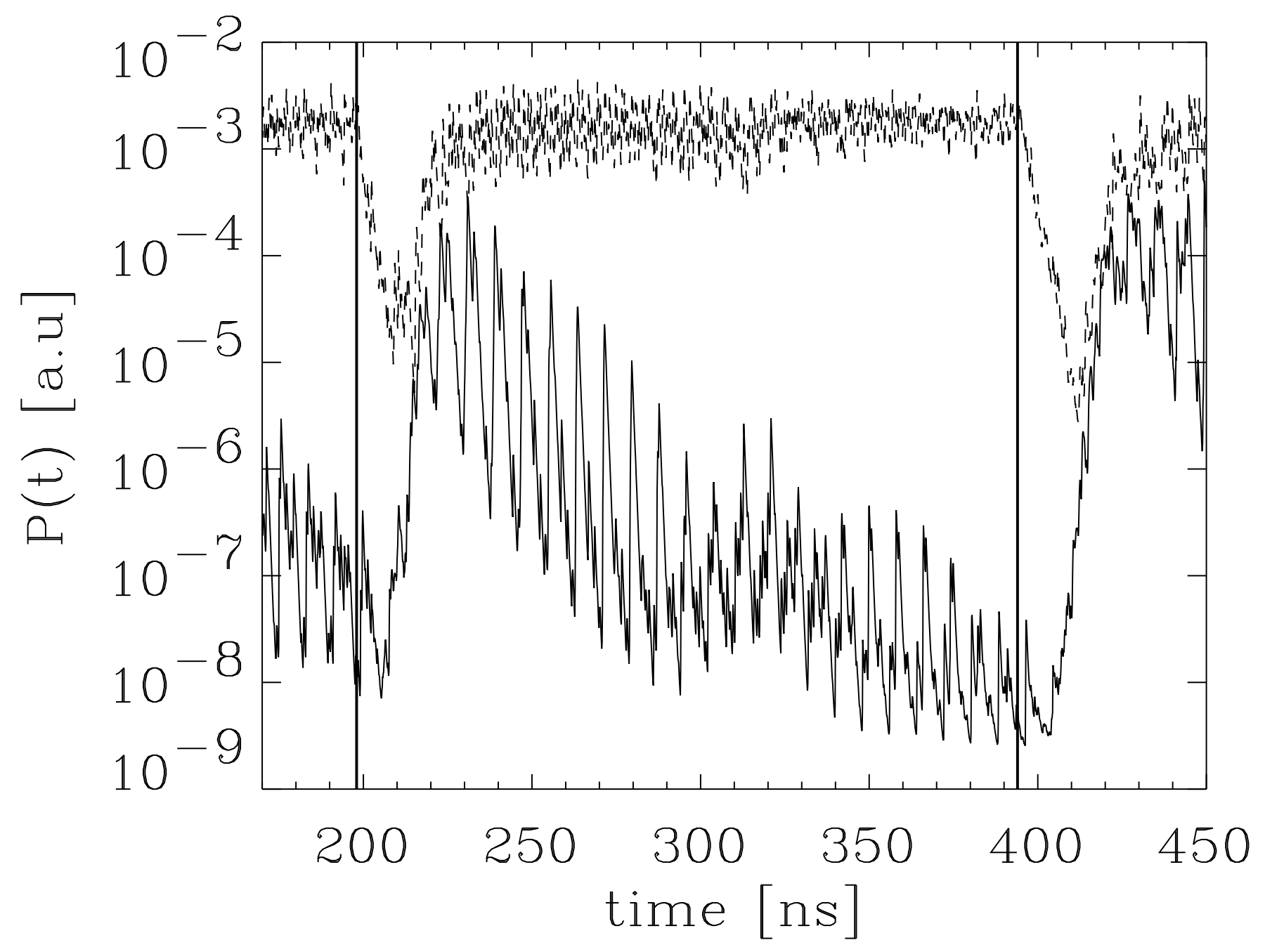

